\title{
STEM-EELS Evaluation of the Dependence of Localized Surface Plasmon Linewidth on the Size of Au Nanoparticles
}

\author{
Jiake Wei ${ }^{1,2}$, Jia Xü ${ }^{3}$, Xuedong Bai ${ }^{2}$ and Jingyue Liu ${ }^{1}$ \\ 1. Department of Physics, Arizona State University, Tempe, Arizona 85287, USA \\ 2. Beijing National Laboratory for Condensed Matter Physics, Institute of Physics, Chinese Academy of \\ Sciences, Beijing 100190, China \\ 3. SEMTE, Arizona State University, Tempe, Arizona 85287, USA
}

Localized surface plasmon (LSP) excitations in metallic nanoparticles (NPs) have recently received increasing attention because of their potential applications in solar energy harvesting, photocatalysis, sensing and biology. Both the LSP resonance energy and peak linewidth depend on the size, shape and environment of the metallic NP, especially for NPs with sizes $<5 \mathrm{~nm}$ [1-2]. With smaller metal NPs quantum size effects, surface diffuse scattering and metal-support interactions can all affect their LSP resonant energies and linewidths. The improved energy resolution of the electron energy loss spectroscopy (EELS) in a monochromated STEM (STEM-EELS) makes it possible to study the intrinsic properties of LSPs in individual small metallic NPs [3]. We report here the STEM-EELS investigation of the dependence of LSP linewidths on the Au NPs in the size range of 4-20 nm in diameter.

The Au NPs were synthesized by reduction of $\mathrm{HAuCl}_{4}$ in $\mathrm{NaBH}_{4}$ solution and were then dispersed onto ultrathin silicon nitride $\left(\mathrm{SiN}_{\mathrm{x}}\right)$ substrate. The STEM-EELS experiments were conducted on a Nion UltraSTEM $^{\mathrm{TM}} 100$ equipped with a monochromator, a C3/C5 aberration corrector and a Gatan Enfina electron energy-loss spectrometer. The EELS spectra were acquired with a dispersion of $5 \mathrm{meV}$ per pixel, a probe convergence semi-angle of $30 \mathrm{mrad}$ and a collection semi-angle of $45 \mathrm{mrad}$, resulting in an energy resolution of $\sim 15 \mathrm{meV}$ in the acquired EELS spectra and significantly reducing the tailing effect of the zero-loss peak on the detection of low-energy loss peaks [4]. The experimental setup is shown in Fig. 1a. The aloof beam condition was used to excite the LSPs in Au NPs.

Figure $1 \mathrm{~b}$ shows a HAADF image of an Au NP of interest and the corresponding EELS spectra acquired at the different positions were shown in Fig. 1c. The LSP resonant peak at $\sim 2.34 \mathrm{eV}$ is clearly revealed when the electron beam was at aloof positions. When the electron beam passed through the center of the $\mathrm{Au}$ NP a prominent broad peak at $\sim 2.44 \mathrm{eV}$ was observed, reflecting the excitation of Au bulk plasmons. All the three spectra in Fig. 1c show non-symmetric profiles, presumably caused by the overlap with the energy loss peak of the inter-band transitions of $\mathrm{Au} 5 \mathrm{~d}$ electrons to the 6sp orbitals. Figure 2a displays a series of EELS spectra, obtained with an aloof beam, of Au NPs with sizes from $\sim 23 \mathrm{~nm}$ to $\sim 5 \mathrm{~nm}$ in diameter. The signal-to-noise ratio of the LSP resonant peak decreases with decreasing particle size but is still good enough for quantitative analysis. The linewidth of the Au LSP resonant peak increases significantly with decreasing particle size. Figure $2 \mathrm{~b}$ plots the dependence of the full-width-at-halfmaximum (FWHM) values of the Au LSP resonant peaks, estimated by fitting the left half of the experimental peak profiles to the corresponding Lorentzian shapes, on the diameter of the Au NPs. When the Au NP size changes from $~ 23 \mathrm{~nm}$ to $\sim 5 \mathrm{~nm}$ the FWHM value of the LSP resonant peak changes from $\sim 0.5 \mathrm{eV}$ to $\sim 1.4 \mathrm{eV}$. Figure 2c plots the FWHM value against the inverse of the particle diameter. We can conclude that for larger particles $(>5 \mathrm{~nm})$, the broadening of the LSP resonant peaks can be considered to be linearly proportional to the inverse of the particle size. For much smaller $\mathrm{Au}$ NPs, the peak broadening, however, seems to increase more rapidly with the decreasing size of the Au 
NPs. The peak broadening mechanisms and the effects of the metal-substrate interaction on the peak broadening are investigated [5].

\section{References:}

[1] JA Scholl et al., Nature, 483 (2012), p. 421.

[2] S Raza et al., Nanophotonics, 2 (2013), p. 131.

[3] J Wei et al., Nano Letters, 15 (2015), p. 5926.

[4] OL Krivanek et al., Nature, 514 (2014), p. 209.

[5] The authors acknowledge the College of Liberal Arts and Sciences of Arizona State University for funding and the use of the John M. Cowley CHREM facilities at Arizona State University.

(a)

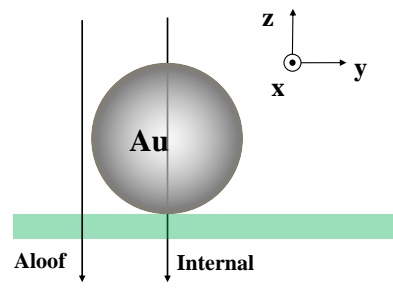

(b)

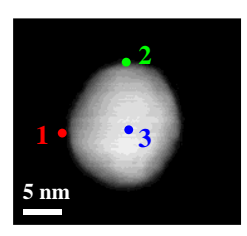

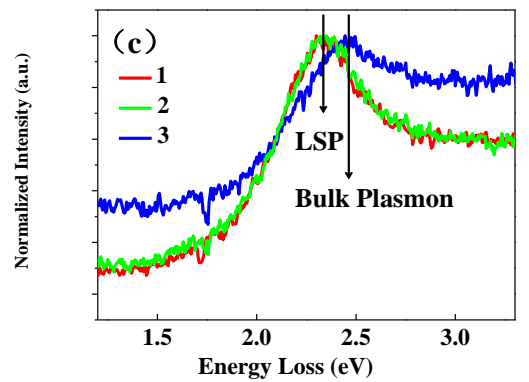

Figure 1. (a) Schematic of the experimental EELS setup; (b) HAADF image of an Au NP and (c) EELS spectra from the Au NP in (b) at different beam positions. Aloof (1,2) and internal (3) beam condition. The EELS spectra were normalized at the peak intensity.
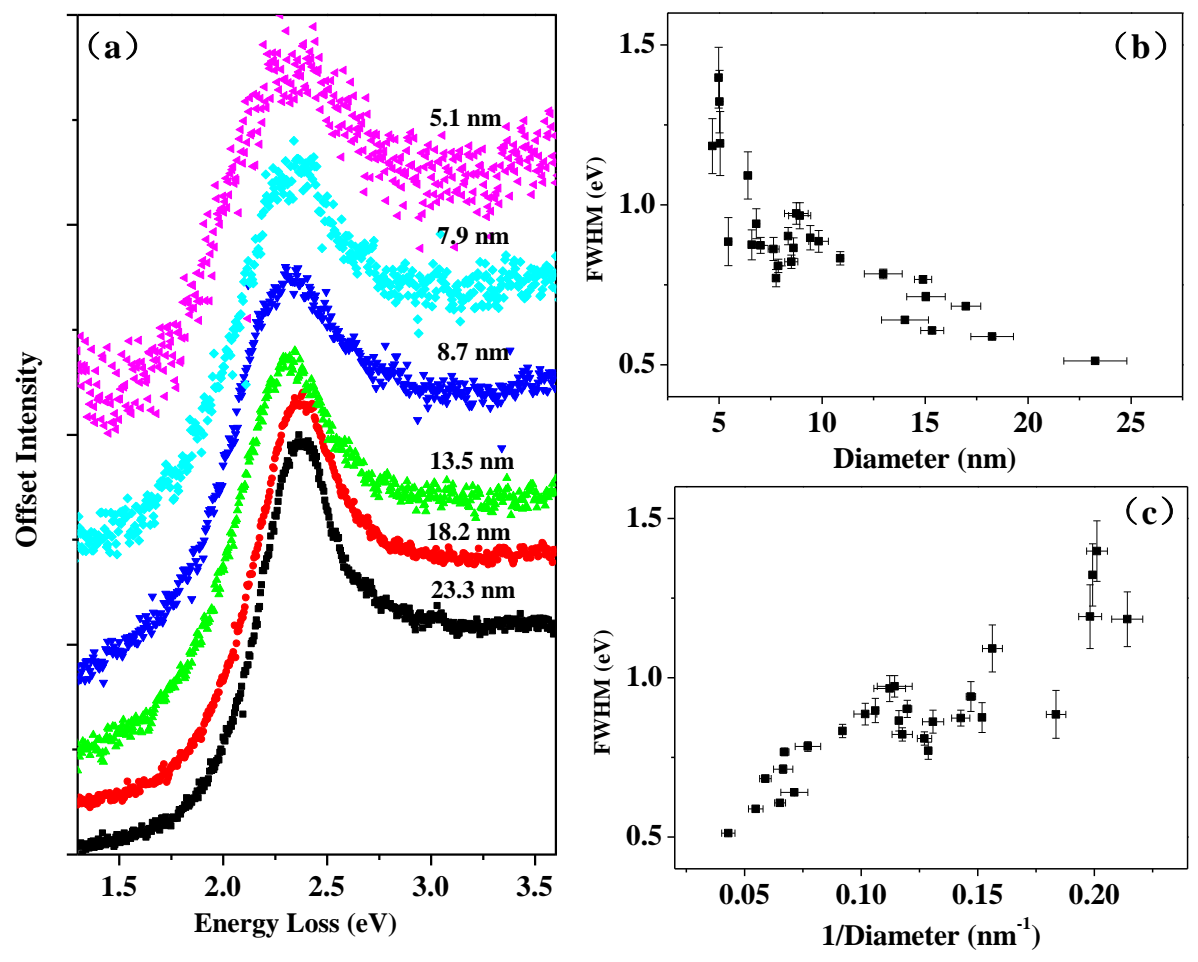

Figure 2. (a) A series of EELS spectra obtained from different sizes of $\mathrm{Au}$ NPs with aloof beam condition. (b) Plot of experimental FWHM values of LSP resonant peak vs. diameter of $\mathrm{Au}$ NP. (c) Plot of FWHM values of LSP resonant peak vs. 1/diameter for $\mathrm{Au}$ NPs of different sizes. The peak broadening becomes much more significant for smaller Au NPs. 